\title{
Percepções sobre o Processo de Trabalho em um Centro de Atenção Psicossocial Infanto-Juvenil
}

\author{
Meyrielle Belotti ${ }^{1}$ \\ Departamento de Terapia Ocupacional e Programa de Pós-Graduação em Psicologia, \\ Universidade Federal do Espirito Santo, Vitória, ES, Brasil \\ Bruna Ceruti Quintanilha \\ Kelly Guimarães Tristão \\ Pedro Machado Ribeiro Neto \\ Programa de Pós-Graduação em Psicologia, Universidade Federal do Espírito \\ Santo, Vitória, ES, Brasil \\ Luziane Zacché Avellar \\ Departamento de Psicologia Social e do Desenvolvimento e Programa de Pós-Graduação \\ em Psicologia, Universidade Federal do Espírito Santo, Vitória, ES, Brasil
}

\begin{abstract}
Resumo
O objetivo da pesquisa foi analisar as percepções dos profissionais de saúde sobre o processo de trabalho e, consequentemente, a produção do cuidado, diante da fusão de um Centro de Atenção Psicossocial Álcool e Drogas infanto-juvenil (CAPS ADi) com um Centro de Atenção Psicossocial Infanto-juvenil (CAPSi), de um município da região sudeste do Brasil. Utilizou-se a abordagem qualitativa. Participaram do estudo 11 profissionais de saúde. Para a coleta de dados foi utilizado a técnica do grupo focal. Os dados foram submetidos à análise de conteúdo. Os resultados indicam que a fusão dos serviços ocorreu de modo verticalizado, não havendo a inclusão dos profissionais no processo de planejamento para a unificação. Esta situação produziu reflexo no trabalho, tais como: dificuldades para a realização do trabalho em equipe sob a ótica da interdisciplinaridade; a construção de um agir descomprometido; e a organização do trabalho por demandas. Além destas questões, verificou-se que a não apropriação dos profissionais dos processos de mudança ocorridos gerou sofrimento nestes.
\end{abstract}

Palavras-chave: Processo de trabalho, serviços de saúde mental, criança, adolescentes.

\section{Perceptions of the Process of Work in a Psychosocial Care Center for Children and Youth}

\begin{abstract}
The aim of this article was to analyze the perceptions of health professionals regarding the process of work and the production of care after the merging of a Psychosocial Care Center for Alcohol and Drug Use for Children and Youth (CAPS ADi) and a Psychosocial Care Center for Children and Youth (CAP$\mathrm{Si}$ ), in a city located in southeastern Brazil. This was achieved using a qualitative approach. The study involved 11 health professionals. Data were collected using focus groups, and examined using content analysis. The results indicated that the merging of the services was implemented in a top-down fashion,
\end{abstract}

1 Endereço para correspondência: Rua Nancy Alves Vieira de Menezes, 50, Mata da Praia, Vitória, ES, Brasil 29.065-560. Fone: (27) 3022-6861 (27) 99949-3596. E-mail: meyri.belotti@gmail.com 
with health professionals excluded from the planning of the merger. This had a significant impact on work performance, leading to difficulties in adopting an interdisciplinary approach to multidisciplinary work; lack of commitment; and a division of the work according to patient needs. Additionally, the exclusion of health professionals from the process of change was identified as a significant cause of distress for these individuals.

Keywords: Work process, mental health services, child, adolescent.

\section{Opiniones en el Proceso del Trabajo en un Centro de la Atención Psicossocial Infanto-Joven}

\section{Resumen}

El objetivo del artículo es analizar las opiniones de los profesionales de salud en el proceso del trabajo e la producción del cuidado, delante de la fusión de uno Centro de la Atención Psicossocial Alcohol y Drogas Infanto-Joven (CAPS Adi) con uno Centro de la Atención Psicossocial Infanto-Joven (CAPSi), de una ciudad de la región del sudeste del Brasil. Se utilizó un enfoque cualitativo. Once profesionales de salud habían participado del estudio. Para la recogida de datos fue utilizado la técnica del grupo focal. Los datos habían sido sometidos al análisis del contenido. Los resultados indican que la fusión de los servicios ocurrió de manera jerárquica, y no hay inclusión de los profesionales en el proceso de planificación para la unificación de los servicios, como: dificultades para llevar a cabo el trabajo en equipo desde la perspectiva de la interdisciplinariedad; la construcción de acciones sin compromisos; y también la organización del trabajo para las demandas. Además, se ha descubierto que la no apropiación de los profesionales en los procesos que se produjeron causaron sufrimiento.

Palabras clave: Proceso del trabajo, servicios de la salud mental, niño, adolescente.

Nos últimos anos, o campo da saúde mental tem passado por mudanças significativas, no que tange os saberes e as práticas, na busca pela superação da lógica manicomial. Estas têm se dado a partir da reestruturação das políticas públicas e da organização da rede de atenção em saúde, o que possibilita a produção de um cuidado ampliado e capaz de atender tanto ao sofrimento psíquico quanto às demandas relacionadas ao uso abusivo de álcool e outras drogas (Ministério da Saúde, 2009a, 2009b, 2009c, 2014).

Neste cenário, a atenção psicossocial passa a ser o modelo norteador do cuidado, sendo este sustentado por um conjunto de novas concepções e conceitos, nos quais se articulam com diversos saberes a respeito do sofrimento psíquico (Luzio \& Sinibaldi, 2012). Assim, esse novo paradigma exige a criação de outras formas de lidar com a experiência da loucura, por meio da reformulação das ações de caráter técnico capazes de: promover a reorganização dos serviços; reconhecer a pessoa em sofrimento psíquico como um ser com história de vida e possuidor de desejos; e de favorecer a criação de estratégias de cuidado na perspectiva da inclusão social, tendo em vista a relação estigmatizada existente em torno da loucura e das pessoas que fazem uso de álcool e outras drogas.

Especificamente, sobre a produção de cuidado em saúde mental infanto-juvenil, cabe destacar que a inclusão dessa temática ocorreu de forma tardia nas políticas públicas da saúde mental, sendo essa constituída pela extensão das práticas voltadas para a população adulta (Lima, 2014). Esse cenário direcionou o cuidado da criança e do adolescente para as instituições totais de natureza privada e filantrópica, que, na sua maioria, atuam de forma fragmentada (Cavalcante, Jorge \& Santos, 2012).

Ressalta-se, também, que a atenção psicossocial é o modelo norteador da produção de cuidado voltado para o público com necessidades decorrentes do uso abusivo de álcool e outras drogas. No entanto, soma-se a esse modelo o uso 
das estratégias de redução de danos. De acordo com o Ministério da Saúde, a redução de danos deve se constituir como um norte ético de todo e qualquer serviço do SUS (Ministério da Saúde, 2009c). Dessa forma, esta se contrapõe ao modelo de atenção pautado exclusivamente na abstinência, logo, visa reduzir os prejuízos de natureza biológica, social e econômica do uso de drogas, respeitando o indivíduo e o seu direito de consumir essas substâncias.

No que se refere às mudanças produzidas na organização dos serviços e na reestruturação das políticas, destaca-se a criação da Rede de Atenção Psicossocial (RAPS), instituída no intuito de ampliar o acesso das pessoas com sofrimento psíquico e com necessidades decorrentes do uso de álcool e outras drogas aos pontos de atenção ${ }^{2}$ - em seus diferentes níveis de complexidade - e de garantir a articulação e integração desses pontos (Ministério da Saúde, 2014). A implantação da RAPS impõe algumas mudanças na configuração dos serviços que integram a rede de saúde mental. Nesse cenário, os CAPSi se caracterizam por prestar assistência às crianças e aos adolescentes com sofrimento psíquico severo e persistentes quanto aos que apresentam necessidade decorrentes do uso de álcool e outras drogas.

No que tange ao processo de trabalho realizado no CAPS, a RAPS reafirma que esse deve ser organizado por meio de uma equipe multiprofissional, que atue sob a ótica da interdisciplinariedade (Ministério da Saúde, 2014). A interdisciplinaridade é uma das formas de interação entre as disciplinas que se caracteriza por apresentar um grau mais avançado de relação, em que se busca a ruptura das fronteiras disci-

\footnotetext{
Principais pontos de atenção da RAPS: Unidades Básicas de Saúde (também chamadas de Postos de Saúde); Núcleos de Apoio à Saúde da Família. Consultórios da rua; Centros de Convivência; Centros de Atenção Psicossocial nas suas diferentes modalidades; Atenção de Urgência e Emergência; Unidades de Acolhimento; Serviços de atenção em regime residencial; Leitos de saúde mental, álcool e outras drogas em Hospitais Gerais e Serviços Residenciais Terapêuticos (Ministério da Saúde, 2014).
}

plinares por meio de complementariedade das áreas de conhecimento (Sousa \& Bastos, 2016). Tavares, Vendrúscolo, Kostulski, e Gonçalves (2012) relatam que há também outras formas de interação entre as disciplinas, tais como: multidisciplinaridade que se constitui pela justaposição de várias disciplinas em torno de um mesmo tema ou problema, não ocorrendo nenhum tipo de articulação entre os profissionais das diferentes áreas de atuação e a transdisciplinaridade que é caracteriza pela horizontalização das relações interdisciplinares, em que há uma integração das disciplinas de um campo particular para uma premissa geral compartilhada, o que implica na construção de um novo campo disciplinar.

Outra mudança, também produzida pela implementação da RAPS, diz respeito à ordenação do cuidado, que passa para a responsabilidade do CAPS ou da Atenção Básica, garantindo o acompanhamento longitudinal do caso e o permanente processo de cogestão (Ministério da Saúde, 2014). A cogestão é uma diretriz ético-política da Política Nacional de Humanização $(\mathrm{PNH})$ que aposta na indissociabilidade entre a produção de cuidado e os modos de gerir os processos de trabalho. Assim, o modelo de cogestão visa à construção dos processos de trabalho organizados através do trabalho em equipe, buscando garantir que o poder seja compartilhado por meio de análises e decisões construídas coletivamente (Ministério da Saúde, 2009a).

Nesta perspectiva, as diretrizes da PNH destacam a necessidade de reorganizar os processos de trabalho para se produzir mudanças na oferta de serviços. Entende-se por processo de trabalho o modo como desenvolvemos nossas atividades profissionais, ou seja, como é realizado o "conjunto de procedimentos pelos quais os homens atuam, por intermédio dos meios de produção, sobre algum objeto para, transformando-o, obterem determinado produto que pretensamente tenha alguma utilidade" (Faria, Werneck, Furquim, Santos \& Teixeira, 2009, p. 21).

Ressalta-se ainda que o processo de trabalho em saúde possui contornos peculiares e a ação do trabalhador demonstra como será realizada a transformação do objeto de trabalho. Assim, o trabalho em saúde não é controlável devido às 
múltiplas necessidades da vida humana (Littike \& Sodré, 2015).

O presente artigo é resultado de uma pesquisa realizada em um CAPSi, situado em um município da região sudeste do Brasil. Sua relevância está no processo singular que a equipe deste serviços vivenciou, pois, o CAPSi em questão passou por uma fusão com outra instituição de saúde, o Centro de Atenção Psicossocial Álcool e Drogas infanto-juvenil (CAPS ADi). Assim, o CAPSi passa a congregar em seu contexto institucional a assistência às crianças e aos adolescentes, tanto com sofrimentos psíquicos severos e persistente, quanto os que apresentam necessidade decorrente do uso abusivo de álcool e outras drogas. Diante desse cenário, o estudo se propõe a analisar as percepções dos profissionais do serviço sobre os efeitos produzidos pela fusão no processo de trabalho deles e, consequentemente, na produção do cuidado.

\section{Método}

Este trabalho tem por objetivo compreender as percepções dos profissionais sobre os efeitos da fusão dos serviços no processo de trabalho e na produção do cuidado com os usuários. Para tanto, a perspectiva da pesquisa qualitativa mostrou-se o método mais apropriado para o que almejávamos investigar. Pesquisas dessa natureza ocupam-se da compreensão dos significados, dos sentidos, das intencionalidades e das questões subjetivas inerentes aos atos, às atitudes, às relações e às estruturas sociais (Minayo, 2012).

Para a coleta de dados utilizou-se da técnica de grupo focal, que visa à interação entre seus participantes, a partir de tópicos previamente definidos pelo pesquisador (Busanello et al., 2013). Nesta proposta, cria-se um ambiente favorável à discussão e que propicie aos participantes manifestarem suas percepções, crenças e atitudes sobre a temática posta em discussão (Minayo, 2012).

A escolha pelo campo se deu pelo fato dos pesquisadores terem parcerias estabelecidas com o local em outros estudos e projetos, o que per- mitiu ter ciência de que o serviço passava pelo processo de fusão com o CAPS ADi. A proposta do estudo foi apresentada na reunião de equipe, em que os profissionais foram convidados a participar dos grupos focais, realizados no referido serviço.

Participaram da pesquisa 11 profissionais: quatro psicólogos, dois assistentes sociais, um técnico de enfermagem, dois terapeutas ocupacionais, um médico e um educador físico. Em função da incompatibilidade de horários, os participantes foram divididos em dois grupos. Foram realizadas duas sessões com cada grupo. Cada sessão teve a duração de aproximadamente uma hora e o intervalo estabelecido para a realização de cada uma foi de 15 dias. Os tópicos eleitos para as discussões foram: (a) o processo de fusão; (b) a organização do serviço a partir da fusão; (c) as dificuldades encontradas nesse processo; (d) a estruturação das estratégias de cuidados diante da nova realidade.

O tratamento do material deu-se por intermédio da análise de conteúdo, conforme proposto por Bardin (2006). A análise de conteúdo configura-se como um conjunto de técnicas de análise das comunicações, que utiliza procedimentos sistemáticos para a descrição do conteúdo das mensagens (Bardin, 2006). Neste estudo, foram realizadas as seguintes etapas: (a) pré-análise, (b) exploração de material e (c) tratamento dos resultados. Na fase da pré-análise foi realizada uma leitura flutuante da transcrição das sessões, sendo recortados trechos das falas que contemplavam os objetivos do estudo. $\mathrm{Na}$ segunda fase foram definidas as categorias com base nas falas selecionadas. Ao total foram obtidas cinco categorias. A última etapa constitui-se na inferência e na interpretação dos dados tendo como elementos norteadores as categorias encontradas.

Para a apresentação de cada categoria foram explicitados extratos das falas dos participantes. As falas foram identificadas por meio da letra $\mathrm{S}$ maiúscula, esta seguida de um número representando os sujeitos, isto é, S1, S2, S3, S4.

A realização da pesquisa foi aprovada pelo Comitê de Ética em Pesquisa com Seres Huma- 
nos (CEP) e pela Secretaria Municipal de Saúde. Todos os participantes assinaram um Termo de Consentimento Livre e Esclarecido, em atendimento à Resolução no 466 (2012) do Conselho Nacional de Saúde.

\section{Resultados e Discussão}

Conforme processo detalhado acima, os dados analisados delinearam cinco categorias: (a) O motivo da fusão dos serviços; (b) O processo de fusão e a organização do trabalho; (c) Os estigmas da loucura e do uso de substâncias psicoativas; (d) O trabalho em equipe; e (e) A produção de cuidado no CAPSi. Será realizada a discussão dos dados concomitantemente a apresentação dos resultados.

\section{O Motivo da Fusão dos Serviços}

Essa categoria incorpora as falas que dizem respeito à compreensão dos participantes sobre as justificativas para a fusão dos serviços. Identificou-se que um dos motivos estava relacionado à inexistência de uma política regulamentadora sobre o CAPS ADi e, também, à ausência de dados que justificassem a sua existência:

"No Brasil tudo isso não existe: CAPS ADi. Ele não está na política. CAPSi é que atende os dois públicos, não faz sentido ter dois serviços diferentes" (S. 3).

"Baseado em que foi criado o CAPS ADi? E baseado em que, que você justifica que ele vai deixar de existir? Entende, não tinha. Então ficava parecendo um tropeço" (S. 7).

Conforme relatado anteriormente, com a implementação da RAPS, os CAPS passam a realizar assistência às pessoas com sofrimento psíquicos severos e persistentes incluindo aquelas com necessidades decorrentes do uso de álcool e outras drogas. Diante desse novo cenário, a criança e o adolescente, que fazem uso dessas substâncias, devem ser assistidas no CAPSi, podendo também serem assistidas nos CAPS AD e CAPS AD III, se estes serviços forem destinados ao público infanto-juvenil; ou seja, apesar de não existir na política a nomenclatura de CAPS ADi, essa demanda pode ser acompanhada no CAPS $\mathrm{AD}$ e no CAPS AD III.
No que se refere à ausência de dados para justificar a existência e/ou manutenção do CAPS ADi, sabe-se que essa situação ocorre devido à inexistência de Sistemas de Informação em Saúde (SIS) específicos em saúde mental e à ausência de um indicador de saúde mental no Sistema de Informação da Atenção Básica (SIAB). Os SIS visam o acompanhamento integral, a avaliação permanente da situação de saúde da população e os resultados das ações executadas, sendo essas informações fundamentais para o acompanhamento, controle e repasse de recursos. O SIAB se constitui em um SIS que auxilia no acompanhamento e na avaliação das atividades realizadas no nível da Atenção Básica (Ministério da Saúde, 2009b).

Neste contexto, a inexistência de um conjunto de dados para nortear o planejamento, o monitoramento, a avaliação e o processo decisório em saúde mental, faz com que os serviços/ ações de saúde mental sejam implementados no "tropeço".

Outro motivo identificado, conforme ilustrado abaixo, diz respeito à percepção dos profissionais, que compunha a rede de saúde do município pesquisado, da necessidade de fortalecimento dos serviços de saúde mental destinada ao público adulto:

Na época que o CAPS ADi foi aberto foi uma coisa assim muito forte... a indignação da rede como um todo: como? Se a gente há anos tá precisando de um segundo CAPS III adulto vocês, vão e abrem um CAPS Adi (S. 9).

Estudos (Couto, 2012; Lima 2014) relatam que a atenção à saúde mental infanto-juvenil, norteada pelos princípios da Reforma Psiquiátrica, ocorreu de forma tardia no Brasil. Além dessa realidade, Couto (2012) ressalta que a produção do cuidado em saúde mental infanto-juvenil permaneceu em segundo plano quando comparado à assistência voltada aos adultos. Segundo Lima (2014), tal situação reforçou o manto da deficiência que encobre a loucura da criança e do adolescente. $\mathrm{O}$ autor ressalta a importância do reconhecimento da loucura nesta faixa etária, para que a saúde mental infanto-ju- 
venil possa sair do marasmo, quando comparada a saúde mental para adultos.

\section{O Processo de Fusão e a Organização do Trabalho}

Essa categoria agrupa falas que estão relacionadas ao modo como ocorreu à fusão dos serviços e seus reflexos na postura dos profissionais de saúde. Verifica-se, nos relatos apresentados, que não houve participação dos trabalhadores no processo de planejamento e de decisão sobre a fusão:

Não houve uma escuta por parte da gestão com as equipes. E foi uma imposição mesmo, eles decidiram, fizeram e ocorreu essa mudança... Num primeiro momento foi muito dificil para as duas equipes. Foi angustiante. Como vai ser? Até hoje ainda existem dificuldades na condução do trabalho (S. 8).

As falas demonstram a fragilidade do modelo de cogestão, uma vez que é possível verificar a predominância de um modelo de gestão vertical, que impossibilitou a apropriação dos profissionais de saúde do seu trabalho. Tal realidade gerou sofrimento nos trabalhadores e produziu dificuldades na organização do trabalho. Morschel e Barros (2014) ressaltam o caráter singular da $\mathrm{PNH}$, visto que sua proposta visa interferir nos processos de formação e de trabalho no campo da saúde. Nesta perspectiva, os autores colocam que essa política se concretiza através de novas formas de organização do trabalho que se contrapõem à lógica dos modos de funcionamento instituídos; ou seja, formas de trabalho que superem o abismo existente entre os que pensam e os que fazem, entre os que planejam e os que executam.

Os profissionais também apontaram que, logo que ocorreu a fusão dos serviços, apesar de estarem no mesmo espaço físico, as demandas ficaram vinculadas aos serviços de origem; ou seja, as crianças e adolescentes com necessidades decorrentes do uso de álcool e outras drogas eram assistidas pelos profissionais que compunham a equipe do CAPS ADi. O contrário também foi verificado, o público com sofrimento psíquico era referenciado para os profissionais que já estavam no CAPSi, quando esse serviço se caracterizava por prestar assistência somente a criança e adolescente com sofrimento psíquico.

“. . . juntou os serviços, mas não misturou..." (S. 10).

“. . . até hoje tem gente que não atende Álcool e Droga. É nítido e notório, o paciente chega ali e: 'Ah, chama fulano, o paciente é dele'. Não se propõem a ir lá" (S. 1).

Pode-se afirmar que o agir descomprometido, ilustrado na fala acima, também é fruto desse modelo de gestão verticalizada. Para Cunha e Magajewski (2012), a inserção dos trabalhados de saúde nos processos de gestão torna-se de fundamental importância para promover a corresponsabilidade no cuidado e para produzir mudanças no modelo de atenção. De modo geral, há um baixo estímulo à inclusão dos profissionais de saúde em espaços de cogestão. Tal realidade reduz as possibilidades para a formação de um pensamento crítico e para a configuração de um novo modo de agir em saúde em que o cuidado passa a ser compartilhado com todos os atores envolvidos.

Acredita-se que, por meio da valorização dos diferentes sujeitos envolvidos com a produção de cuidado que é possível reafirmar as diretrizes do SUS. Ao mesmo tempo, garante-se a motivação, o estímulo à reflexão e a criatividade, bem como, minimiza-se o sofrimento do trabalhador de saúde através da promoção da autoestima (Cunha \& Magajewski, 2012).

\section{Os Estigmas da Loucura e do Uso de Álcool e outras Drogas}

As conotações sociais presentes em torno da loucura e das crianças e adolescentes com necessidades decorrentes do uso de álcool e outras drogas foi outro aspecto que emergiu nas falas apresentadas. $\mathrm{Na}$ análise dos dados, identificou-se a dificuldade de vinculação dos usuários do antigo CAPS ADi ao CAPSi, em função da construção social acerca da loucura, calcado no preconceito/rejeição:

"Ainda tem essa questão do tipo: não quero ir ao CAPS porque é lugar de doido" (S. 2).

Apesar dos esforços dirigidos à mudança na produção de cuidado e às estratégias de inclusão 
social de pessoas com sofrimento psíquico, verifica-se que a construção acerca da loucura ainda se encontra estigmatizada. Os processos de estigmatização se constituem como um dos entraves para o avanço da construção de um outro lugar social para o "louco". Para tanto, torna-se necessário estabelecer novas relações com o sujeito reconhecido como louco (Belotti \& Lavrador, 2016). Ou seja, é preciso proporcionar sua circulação pelos diferentes espaços da cidade; possibilitar condições de moradia, de educação e de emprego; incentivar a organização coletiva dos usuários de saúde mental e seus familiares via criação de cooperativas e de associações, dentre outras ações.

Também foi possível identificar a conotação negativa associada à criança e ao adolescente com necessidades decorrentes do uso de álcool e outras drogas. Verifica-se, nas falas apresentadas abaixo, que esses são estigmatizados como perigosos e como aqueles que exercem influência "negativa" no outro, com a intenção de promover o uso de algum tipo de droga em outrem. Por consequência, há um desejo de distanciamento social desses sujeitos:

"Algumas mães com filhos com transtorno que viam alguns adolescentes usuários de $A D$ ficavam assustadas. 'Pera ai? Agora esses meninos vão estar aqui também? Afasto meu filho das drogas, agora vejo o menino aqui dentro?" (S. 5).

De acordo com Ronzani e Andrade (2014) diversas razões podem favorecer o processo de estigmatização das pessoas com necessidades decorrentes do uso abusivo de álcool e outras drogas. Dentre estas, os autores destacam o fato desses usuários serem vistos como alguém com baixa autoestima e com fraqueza de caráter, sendo ele o único responsável pelo seu problema. Além desses "rótulos", verifica-se que a estigmatização desse público está associada à existência de uma forte conotação moral, o que dificulta a realização de abordagens "adequadas" (Ronzani \& Andrade, 2014).

Apesar de verificar a existência das relações estigmatizadas, no que concerne ambas as demandas presentes no CAPSi, a análise dos dados também aponta que a convivência foi um fator que favoreceu a redução das conotações negativas; tanto acerca da loucura quanto das crianças e dos adolescentes com necessidades decorrente do uso de álcool e outras drogas:

“. . . depois as mães mesmo reconheceram que não era assim. Que os usuários de álcool e outras drogas também precisam de cuidado" (S. 10).

".. . com o passar do tempo eles começaram a entender e respeitar o modo de funcionamento dos usuários com transtorno" (S. 9).

Rüsch, Angermeyer e Corrigan (2005) apontam que o contato e a interação com as pessoas estigmatizadas é uma das estratégias para a redução do estigma. Os autores enfatizam, também, a importância da criação de ações educativas, no sentido de promover a substituição dos mitos por informações e de protesto, que visam à mobilização social para suprimir as atitudes e comportamentos estereotipados.

Ressalta-se ainda que, promover estratégias que visam à redução do estigma envolve, necessariamente, a realização de intervenções no nível macro, como por exemplo, a implementação de políticas públicas. Entretanto, a desmistificação em torno da loucura e das pessoas com necessidade decorrentes do uso de álcool e outras drogas, não pode ser pensada somente em termos macro. É preciso desenvolver ações no plano micro, ou seja, no cotidiano das instituições e nas suas práticas, que sejam capazes de produzir mudanças significativas na vida dessas pessoas.

\section{O Trabalho em Equipe}

As falas evocadas relacionadas às formas de relações interdisciplinares e trabalho em equipe foram agrupados nesta categoria. No que se refere às interações interdisciplinares estabelecidas entre os profissionais, observa-se uma contradição nos relatos. Algumas falas demonstram que o trabalho em equipe é organizado por turnos de trabalho, configurando-se diferentes equipes em um mesmo serviço. Outros profissionais colocam que as relações entre as disciplinas se modificam a cada momento:

"tem três equipes aqui dentro. Não é porque juntou todo mundo que se tornou uma equipe. Então tem o grupo da manhã, o grupo da tarde 
e nós que somos o meio termo, que fazemos $8 h$ " (S. 8).

"temos que ser só trans ou só multi? Vamos ser essa metamorfose ambulante..." (S. 3).

Como relatado anteriormente, os modos de organização do trabalho em equipe de saúde, suas relações e interações estabelecidas entre as disciplinas, pode ocorrer de formas diferenciadas. Por um lado, temos multidisciplinaridade e a pluridisciplinaridade, com quase nenhum contato entre as disciplinas, e, no outro extremo, temos a interdisciplinaridade e transdisciplinaridade com uma grande interação entre elas (Sousa \& Bastos, 2016; Tavares et al., 2012).

Na primeira fala verifica-se a existência de três equipes no CAPSi, que estabelecem formas de relação diferenciadas com os seus membros, porém não estabelecem interações entre elas. Já a segunda fala caracteriza o trabalho em equipe como uma "metamorfose ambulante", em que a organização do trabalho oscila entre multi, inter e transdisciplinaridade. Isto relaciona-se aos momentos vivenciados pela equipe:

Às vezes tem momentos que a gente está eufórico. As coisas avançando, caminhando. A gente acha que dali só vai mais ainda, como maníaco acha. Tem momentos que a gente está assim: pra baixo, desanimado, desacreditado. E acha que vai piorar mais ainda. Então é assim, essas coisas comparecem em uma equipe. A equipe nem sempre está num rumo, pra melhora. E nem sempre ela está acabada, esfacelada. Cabe a gente saber lidar com esses momentos de uma equipe (S. 1).

Os dados indicam que também há necessidade de se preservar os núcleos de competência, sem perder de vista a flexibilização e o compartilhamento do conhecimento:

Estou indo no saber dele, permitindo que ele também se aproprie do meu. Mas sem atropelar o dele e que ele atropele o meu. Até onde posso ir com meu saber sem atropelar o saber do meu colega? Onde posso deixar o saber dele entrar na minha práti$c a$ ? (S. 5).

Entende-se o núcleo de competências como um conjunto de práticas e saberes específicos de cada profissional, que proporcionam a autonomia desses, mas ao mesmo tempo produzem interdependência (Sousa \& Bastos, 2016). Neste contexto, os núcleos de competência, isoladamente, não conseguem dar conta das necessidades do campo da saúde, sendo necessária a criação de mecanismos de flexibilidade entre os limites das competências para proporcionar um cuidado integral.

As questões subjetivas que se referem ao cotidiano do trabalho em equipe, também se fizeram presentes nesta categoria. Verificou-se que essas questões influenciam na construção dos processos de trabalho:

É uma construção. Que envolve aquilo que há de melhor e pior no ser humano: vaidade, egoísmo, narcisismo, ódio, inveja. Quando a gente se reuni, isso tudo está colocado. Como construir isso no sentido que se transforme numa ferramenta de trabalho (S. 4).

A subjetividade, conforme proposto por Nardi (2006), é um conceito estratégico para a psicologia social na medida em que compreende a indissociabilidade entre individual e coletivo, dentro e fora, indivíduo e sociedade. Neste contexto, o autor ressalta que os processos de trabalho em saúde não podem ser analisados apenas pela vertente técnica da produção, mas considerando também o modo como os sujeitos vivenciam e dão sentido às suas experiências de trabalho.

Franco e Merhy (2012) colocam que os processos de trabalho em saúde, na sua micropolítica, revelam um mundo dinâmico, criativo, não estruturado e com alta possibilidade inventiva; ou seja, o cuidado é uma produção social e também subjetiva. Diante desta perspectiva, os autores reconhecem que a vida produtiva é organizada pelas relações e que o agir cotidiano dos trabalhadores é permeado pelas relações estabelecidas entre esse e pelo cenário que se encontra. Assim, o trabalho em equipe e, consequentemente, a produção de cuidado estão colocados em um território composto por múltiplas determinações, em que por meio dos encontros e das negociações dos sentidos podem ou não produzir um agir coerente. 


\section{A Produção de Cuidado no CAPSi}

As questões referentes à clínica e a produção de cuidado também emergiram nas falas apresentadas. Conforme ilustrado nos relatos abaixo, os profissionais identificam algumas distinções na condução da clínica da criança e do adolescente com sofrimento psíquico com necessidades decorrentes do uso de álcool e outras drogas:

Qual o ponto de junção das duas clinicas, isso é desafiador. Acho que não houve essa sensibilidade por parte da área técnica, no sentido de sensibilizar a gestão de que a característica da clinica de AD é diferente da clinica do transtorno. Como conciliar as clinicas é o grande desafio (S. 6).

São clínicas distintas, mas tem muitas coisas em comum né. No sentido assim, a questão da inserção social, da escola. As questões familiares né, as complexidades são muito parecidas, a gente tem muita coisa em comum, a condução clínica que é distinta, no sentido assim, do um a um ali, isso é distinto mesmo (S. 11).

Como já relatado anteriormente, a produção de cuidado em saúde mental é norteada pelo modelo da atenção psicossocial. Sendo acrescidas as estratégias de redução de danos nas demandas vinculadas ao uso de álcool e outras drogas. No que se refere à temática da saúde mental infanto-juvenil, soma-se a essas propostas, a necessidade de contemplar o sistema de particularidade em torno da criança e do adolescente, que envolve a inclusão na produção de cuidado de outros setores: atenção básica, educação, assistência social, justiça e direitos (Couto, 2012; Lima, 2014; Ministério da Saúde, 2014). Além dessa particularidade, ressalta-se também a necessidade de incluir a família no tratamento; de adequar a linguagem e de inserir o lúdico no campo das intervenções (Avellar \& Bertollo, 2008; Guerra, 2005).

Assim, "o ponto de junção" da clínica direcionada à criança e ao adolescente com sofrimento psíquico com a clínica voltada para as demandas decorrentes do uso de álcool e outras drogas, seria tanto o modelo de atenção psicossocial quanto o sistema de particularidade da saúde mental infanto-juvenil. O que as diferencia, no sentido de "um a um", seria o uso da estratégia de redução de danos no cuidado da criança e do adolescente com necessidades decorrentes do uso abusivo de álcool e outras drogas.

Outra diferença identificada na produção do cuidado refere-se à forma de utilização do serviço. Verifica-se que as crianças e adolescentes com necessidades decorrentes do uso de álcool e outras drogas vão ao serviço, principalmente, para suprir suas necessidades básicas, sendo estes momentos utilizados pelos profissionais para a realização de intervenções:

porque essa clientela [crianças e adolescentes que fazem uso do álcool e outras drogas], eles vem sem agenda, eles vem solicitando: "ah quero lanchar. Quero almoçar. Eu preciso de um banho". Eles vêm só pra isso. Ai a partir disso, a gente conversa, vai fazendo as intervenções com eles, criando um vínculo (S. 7).

Observa-se na fala uma ruptura com a clínica tradicional e a criação de novas metodologias de organização do trabalho. Para Sousa (2010), o CAPS tem como proposta a construção de uma clínica cada vez mais fluida e diversificada para intervir em situações complexas, nas quais o sistema tradicional se mostra insuficiente. Para tanto, a estrutura física desses serviços deve ser diferenciada, permitindo uma maior circulação e uso do espaço tanto pelos usuários quanto pelos profissionais. Neste contexto, embora a existência de um espaço de escuta reservado seja necessária, a clínica pode ser realizada nas mais diversas situações, se caracterizando com inovadora e inventiva.

\section{Considerações Finais}

Este artigo teve como objetivo analisar as percepções dos profissionais de saúde sobre os efeitos produzidos no processo de trabalho e, consequentemente, na produção do cuidado, diante da fusão de um CAPS ADi com um CAPSi. Os resultados indicam que a não participação dos profissionais no planejamento das ações para a unificação dos serviços produziu reflexo no trabalho. Dentre esses, verificou-se dificul- 
dades para: a realização do trabalho em equipe multidisciplinar, sob a ótica da interdisciplinaridade; a construção de um agir comprometido; e a divisão de trabalho por demandas. Alguns profissionais só atendiam demandas relacionadas ao sofrimento psíquico e outros as decorrentes do uso de álcool e outras drogas.

Ressalta-se também, a exclusão dos usuários e seus familiares no processo de planejamento para a fusão dos serviços. Verifica-se que esta realidade aponta para mais uma fragilidade do modelo de cogestão, visto que tal política também propõem o fortalecimento da participação social, por meio da inclusão dos usuários no planejamento, implementação e avaliação dos processos de produção de saúde (Ministério da Saúde, 2009a). Destaca-se ainda que, em relação aos estigmas apresentados pelos usuários e seus familiares, pode-se aferir que essas conotações sociais teriam sido desmistificadas mais facilmente se estes estivessem incluídos nas discussões e ações de planejamento para a junção do serviço. No entanto, conforme relatado no item resultados e discussões, verificou-se que a convivência entre ambas as demandas presente no CAPSi, favoreceu a diminuição dos estigmas em torno da criança e do adolescente com sofrimento psíquico e com necessidades decorrentes do uso do álcool e outras drogas.

Diante do exposto, pondera-se que as dificuldades identificadas no processo de trabalho do serviço estudado poderiam ter sido amenizadas se os diferentes sujeitos implicados com a produção de cuidado estivessem envolvidos nas discussões relacionadas a fusão dos serviços; ou seja, se em vez de uma postura verticalizada, tivessem sido criados espaços que favorecessem a problematização do processo de trabalho diante de uma nova possibilidade de realidade institucional. Tais espaços de discussão poderiam favorecer a corresponsabilização na produção de cuidado, promover uma melhor interação entre os profissionais e, consequentemente, facilitar a organização do trabalho em equipe, além de fortalecer o vínculo entre usuários, familiares e profissionais.

É importante salientar que, neste estudo não foi possível inserir, na coleta de dados, os profissionais da gestão que discutiram e decidiram sobre a fusão do CAPS ADi com o CAPSi, e os usuários e seus familiares. Não sendo possível, analisar sobre a ótica destes como ocorreu o processo de junção.

\section{Referências}

Avellar, L. Z., \& Bertollo, M. (2008) A saúde mental na infância e adolescência e o diálogo necessário entre as dimensões clínica, ética e política. In E. M. Rosa, L. Souza, \& L. Z. Avellar (Eds.), Psicologia Social: Temas em debate (pp. 68-87). Vitória, ES: Universidade Federal do Espírito Santos.

Bardin, L. (2006). Análise de conteúdo (L. de A. Rego \& A. Pinheiro, Trads.). Lisboa: Edições 70.

Belotti, M., \& Lavrador, M. C. C. (2016). A prática do apoio matricial e os seus efeitos na Atenção Primária à Saúde. Cadernos de Terapia Ocupacional - UFSCar, 24(2), 373-378. doi:10.4322\%2F0104-4931.ctoRE0627

Busanello, J., Lunardi, W. D., Filho, Kerber, N. P. C., Santos, S. S. C., Lunardi, V. L., \& Pohlmann, F. C. (2013). Grupo focal como técnica de coleta de dados. Cogitare Enfermagem, 18(2), 358-364. doi:10.5380/ce.v18i2.32586

Cavalcante, C. M., Jorge, M. S. B., \& Santos, D. C. M. dos. (2012). Onde está a criança?: Desafios e obstáculos ao apoio matricial de crianças com problemas de saúde mental. Physis: Revista de Saúde Coletiva, 22(1), 161-178. doi:10.1590/ S0103-73312012000100009

Couto, M. C. V. (2012). Politica de Saúde Mental para crianças e adolescentes: Especificidades e desafios da experiência brasileira (2001-2010) (Tese de doutorado, Instituto de Psiquiatria, Universidade Federal do Rio de Janeiro, RJ, Brasil).

Cunha, P. F., \& Magajewski, F. (2012). Gestão participativa e valorização dos trabalhadores: Avanços no âmbito do SUS. Saúde e Sociedade, 21(Suppl. 1), 71-79. doi:10.1590/S010412902012000500006

Faria, H. P., Werneck, M. A. F., Furquim, M. A., Santos, M. A., \& Teixeira, P. F. (2009). Processo de trabalho em saúde (2. ed.). Belo Horizonte, MG: Nescon. 
Franco, T. B., \& Merhy, E. E. (2012). Cartografias do Trabalho e Cuidado em Saúde. Revista Tempus Actas de Saúde Coletiva, 6(20), 151-163. doi:10.18569/tempus.v6i2.1120

Guerra, A. M. C. (2005). A psicanálise no campo da saúde mental infanto-juvenil. Psyché, 9(15), 139-154. Recuperado em http://pepsic.bvsalud. org/scielo.php?script=sci_arttext\&pid=S1415$11382005000100011 \& \operatorname{lng}=\mathrm{pt \& nrm}=\mathrm{i} \& \operatorname{lng}=\mathrm{pt}$

Lima, R. C. (2014). Saúde mental na infância e adolescência. In M. A. S. Jorge, M. C. de A. Carvalho, \& P. R. F. Silva (Eds.), Politicas e Cuidados em Saúde Mental: Contribuições para a prática profissional (pp. 229-254). Rio de Janeiro, RJ: Fundação Oswaldo Cruz.

Littike, D., \& Sodré, F. (2015). Arte do Improviso: O processo de trabalho dos gestores de um hospital universitário federal. Ciência \& Saúde Coletiva, 20(10), 3051-3062. doi:10.1590/1413812320152010.00042015

Luzio, C. A., \& Sinibaldi, B. (2012). Atenção Psicossocial e Psicologia: Um mapeamento da produção científica. Revista de Psicologia da UNESP, 12(2), 99-110. Recuperado em http://seer.assis. unesp.br/index.php/revpsico/article/view/27/24

Minayo, M. C. de S. (2012). Análise qualitativa: Teoria, passos e fidedignidade. Ciência \& Saúde Coletiva, 17(3), 621-626. doi:10.1590/S141381232012000300007

Ministério da Saúde. (2009a). Política Nacional de Humanização da Atenção e Gestão do SUS. Brasília, DF: Autor. Recuperado em http://bvsms. saude.gov.br/bvs/publicacoes/humaniza_sus atencao basica.pdf

Ministério da Saúde. (2009b). A experiência brasileira em sistemas de informação em saúde. Brasília, DF: Autor. Recuperado em http:// bvsms.saude.gov.br/bvs/publicacoes/experiencia_brasileira_sistemas_saude_volume1.pdf

Ministério da Saúde. (2009c). Portaria 1.190, de 4 de junho de 2009. Institui o Plano Emergencial de Ampliação do Acesso ao Tratamento e Prevenção em Álcool e outras Drogas no Sistema Único de Saúde - SUS (PEAD 2009-2010) e define suas diretrizes gerais, ações e metas. Brasília, DF: Autor. Recuperado em http:// bvsms.saude.gov.br/bvs/saudelegis/gm/2009/ prt1190_04_06_2009.html

Ministério da Saúde. (2014). Atenção psicossocial a crianças e adolescentes no SUS: Tecendo redes para garantir direitos (Versão preliminar). Brasília, DF: Autor. Recuperado em http://bvsms. saude.gov.br/bvs/publicacoes/atencao psicossocial_criancas_adolescentes_sus.pdf

Morschel, A., \& Barros, E. B. (2014). Processos de trabalho na saúde pública: Humanização e efetivação do Sistema Único de Saúde. Saúde e Sociedade, 23(3), 928-941. doi:10.1590/S010412902014000300016

Nardi, H. C. (2006). Ética, trabalho e subjetividade: Trajetórias de vida no contexto do capitalismo contemporâneo. Porto Alegre, RS: Universidade Federal do Rio Grande do Sul.

Resolução $n^{\circ} 466$ de 12 de dezembro de 2012. (2012). Regulamenta pesquisas em seres humanos no Brasil e da outras disposições. Brasília, DF: Conselho Nacional de Saúde. Recuperado em http://conselho.saude.gov.br/resolucoes/2012/Reso466.pdf

Ronzani, T. M., \& Andrade, T. M. (2014). A estigmatização associada ao uso de substâncias como obstáculo à detecção, prevenção e tratamento. In Uso de substâncias psicoativas no Brasil (5. ed.). Brasília, DF: Secretaria Nacional de Políticas sobre Drogas.

Rüsch, N., Angermeyer, M. C., \& Corrigan, P. W. (2005). Mental illness stigma: Concepts, consequences and initiatives to reduce stigma. $E u$ ropean Psychiatry, 20, 529-539. doi:10.1016/j. eurpsy.2005.04.004

Sousa, P. S. M. (2010). A inserção da psicanálise na saúde mental da reforma psiquiátrica brasileira: Possibilidades e desafios (Dissertação de mestrado, Programa de Pós-Graduação em Psicologia Clínica e Cultura, Universidade Federal de Brasília, DF, Brasil).

Sousa, I. F. de, \& Bastos, P. R. H. de O. (2016). Interdisciplinaridade e formação na área de farmácia. Trabalho, Educação e Saúde, 14(1), 97-117. doi:10.1590/1981-7746-sip00092

Tavares, S. O., Vendrúscolo, C. T., Kostulski, C. A., \& Gonçalves, C. dos S. (2012). Interdisciplinaridade, multidisciplinaridade ou transdisciplinaridade. In Anais do $5^{\circ}$ Interfaces no fazer psicológico. Santa Maria, RS: Centro Universitário Franciscano. Recuperado em http://www.unifra. br/eventos/interfacespsicologia/consulta_anais. asp

Recebido: 03/11/2015

$1^{a}$ revisão: 07/07/2016

$2^{a}$ revisão: $18 / 07 / 2016$

Aceite final: 03/08/2016 